Conclusions FW PSIR LGE significantly increases sub-epicardial LGE detection in patients with suspected myocarditis compared to standard bright blood LGE and importantly changes the clinical diagnosis in a third of patients.

\section{QUANTITATIVE CMR MYOCARDIAL PERFUSION MAPPING TO ASSESS HYPERAEMIC RESPONSE TO ADENOSINE STRESS: A NEW REFERENCE STANDARD?}

Tushar Kotecha, Juan Manuel Monteagudo, Ana Martinez-Naharro, Callum Little, Liza Chacko, James Brown, Daniel Knight, Philip Hawkins, Hui Xue, Peter Kellman, Niket Patel, Tim Lockie, Roby Rakhit, Marianna Fontana. UCL Department of CMR, Royal Free London NHS Foundation Trust, London, UK

\subsection{6/heartjnl-2019-BSCMR.18}

Background Assessment of hyperaemia during adenosine stress CMR remains a clinical challenge with lack of a gold standard marker of adequate stress. Multiple parameters including the splenic switch off (SSO) sign, heart rate response (HRR) and blood pressure response (BPR) are used in clinical practice. Perfusion mapping provides a pixelwise representation of myocardial blood flow (MBF), allowing for measurement of $\mathrm{MBF}$ at a regional level. This may provide an alternative tool for assessment of hyperaemia.

Objectives To validate the use of stress MBF for assessment of hyperaemic response and compare this to currently used clinical markers.
Methods In total, 216 subjects were recruited. This included 3 cohorts: 1) Derivation cohort (22 healthy volunteers) to identify a stress MBF threshold value representative of the normal minimum response to adenosine; 2) Validation cohort (37 patients with suspected coronary disease) who underwent stress CMR and invasive coronary physiological assessment on the same day, to validate the stress MBF threshold value against invasive markers of hyperaemia; 3) Clinical cohort (159 patients undergoing clinically-indicated adenosine stress CMR) to assess the presence of stress MBF-defined hyperaemia and other physiological markers of hyperaemia (SSO, HHR and BPR).

Results From the derivation cohort, maximum stress MBF (SMBFmax) $>1.43 \mathrm{ml} / \mathrm{g} / \mathrm{min}$ was derived as the threshold value of hyperaemia (defined as 1.96 standard deviations below the sample mean of lowest stress MBF values). This threshold was tested in the validation cohort: $100 \%$ of patients with invasive evidence of hyperaemia demonstrated SMBFmax $>1.43 \mathrm{ml} / \mathrm{g} / \mathrm{min}, 81 \%$ had $\mathrm{SSO}$ and $81 \%$ had HRR >10 bpm. Of the clinical cohort, 93\% had hyperaemia defined by SMBFmax compared to $71 \%$ using SSO and $81 \%$ using HRR. SMBFmax was no different in those with or without SSO $\quad(2.58 \pm 0.89 \mathrm{ml} / \mathrm{g} / \mathrm{min}$ vs $2.54 \pm 1.04 \mathrm{ml} / \mathrm{g} / \mathrm{min}$, $\mathrm{p}=0.84$ ) but those with HRR had significantly higher SMBFmax $(2.69 \mathrm{ml} / \mathrm{g} / \mathrm{min}$ vs $1.95 \mathrm{ml} / \mathrm{g} / \mathrm{min}, \mathrm{p}<0.001)$.

HRR >16 bpm was able to predict SMBFmax $>1.43 \mathrm{ml} / \mathrm{g} /$ min with sensitivity $63 \%$ and specificity 91\% (AUC 0.87 , $\mathrm{p}<0.001$ ) and performed better than SSO (AUC 0.62, $\mathrm{p}<0.001$ for comparison of methods).
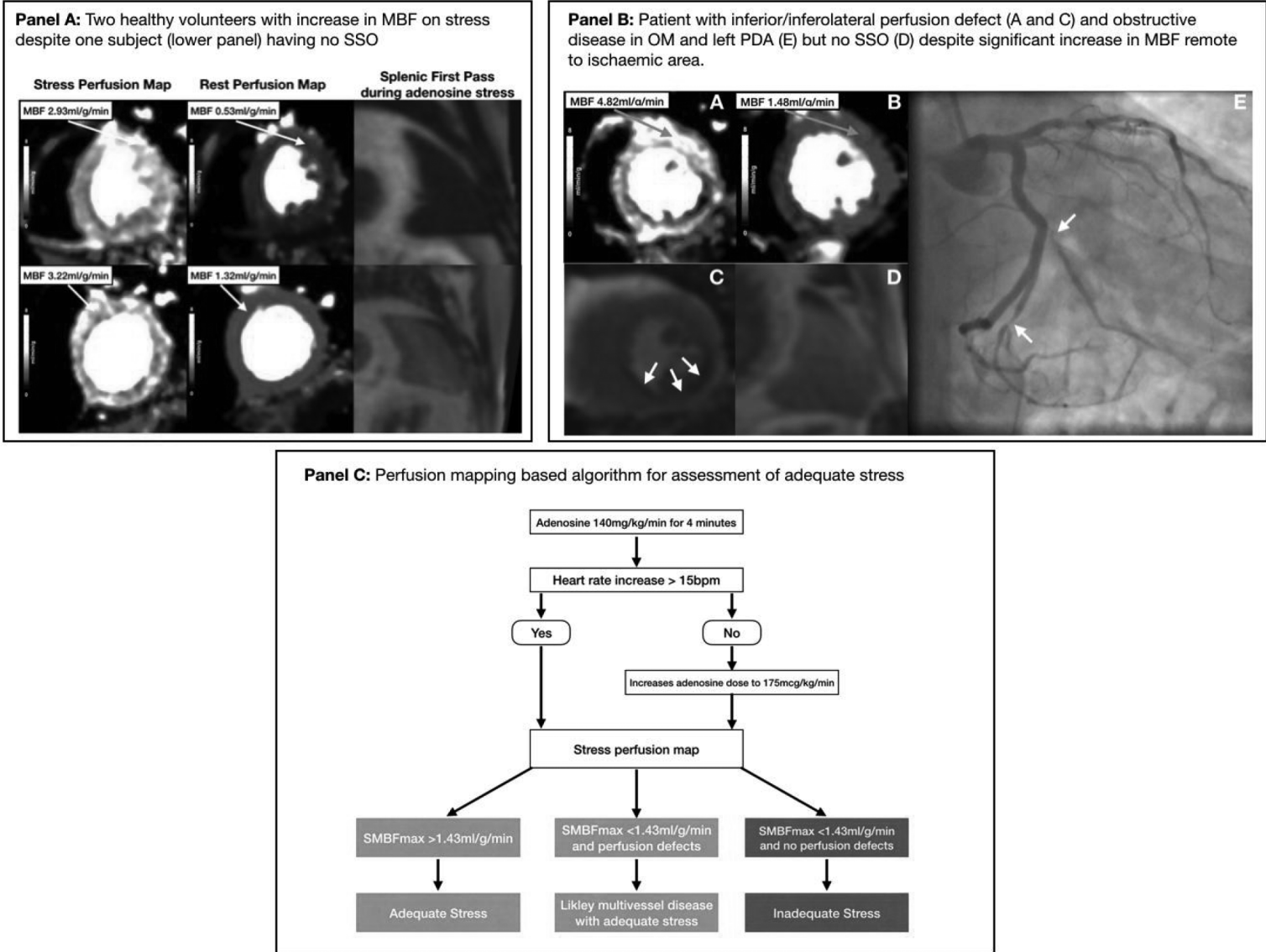
Conclusion Adenosine-induced increase in MBF measured using perfusion mapping is accurate for the confirmation of hyperaemia during stress CMR studies and is superior to traditional, clinically used markers of adequate stress.

\section{MYOCARDIAL PERFUSION MAPPING IN CARDIAC AMYLOIDOSIS- UNEARTHING THE SPECTRUM FROM INFILTRATION TO ISCHAEMIA}

${ }^{1}$ Tushar Kotecha, 'Ana Martinez-Naharro, ${ }^{1} J a m e s$ Brown, ${ }^{1}$ Callum Little, 'Daniel Knight, ${ }^{1}$ Alexandros Steriotis, ${ }^{1}$ Niket Patel, ${ }^{1}$ Roby Rakhit, ${ }^{1}$ Philip Hawkins, 'Julian Gillmore, ${ }^{2}$ James Moon, ${ }^{3}$ Hui Xue, ${ }^{3}$ Peter Kellman, ${ }^{1}$ Marianna Fontana. ${ }^{1}$ National Amyloidosis Centre, Division of Medicine, University College London, Royal Free Hospital, London, UK; ${ }^{2}$ Barts Heart Centre, West Smithfield, London, UK; ${ }^{3}$ National Heart, Lung and Blood Institute, National Institutes of Health, Bethesda, Maryland, USA

\subsection{6/heartjnl-2019-BSCMR.19}

Background Cardiac involvement is the main driver of outcome in systemic amyloidosis, but the relationship between amyloid deposits and outcomes is not well understood. The simple explanation of physical, mechanical replacement of the interstitium by amyloid seems insufficient. Preliminary studies support the hypothesis that myocardial ischaemia could contribute to cell damage.

Purpose (1) To assess myocardial ischaemia in cardiac amyloidosis. (2) To compare patients with cardiac amyloidosis to patients assessed on invasive coronary angiography (ICA) to have normal coronary physiology (NCP), microvascular dysfunction (MVD) and triple vessel coronary disease (3VD). (3) To assess correlation of perfusion mapping to markers of disease severity and prognosis.

Methods 86 patients and 20 healthy volunteers (HV) underwent CMR at $1.5 \mathrm{~T}$ (Siemens) with standard cine, PSIR-LGE, T1,T2,Extracellular Volume (ECV) mapping and adenosine stress with myocardial blood flow (MBF) mapping. Thirtyeight patients also underwent ICA with 3 vessel assessment of Index of Microcirculatory Resistance and Fractional Flow Reserve: 7 had cardiac amyloidosis, 8 had NCP, 15 had MVD and 8 had 3 VD.

Results Cardiac amyloidosis patients had severe reduction in stress MBF and myocardial perfusion reserve (MPR) $(1.22 \mathrm{ml} /$ $\mathrm{g} / \mathrm{min} \pm 0.70$ and $1.62 \pm 0.63)$ compared to $\mathrm{HV}(3.21 \mathrm{ml} / \mathrm{g} / \mathrm{min}$ $\pm 0.64, \mathrm{p}<0.001$ and $4.17 \pm 0.78, \mathrm{p}<0.001)$, NCP $(2.66 \pm 0.56$, $\mathrm{p}<0.001$ and $2.51 \pm 0.43, \mathrm{p}=0.036)$ and MVD $(2.10 \pm 0.31$,

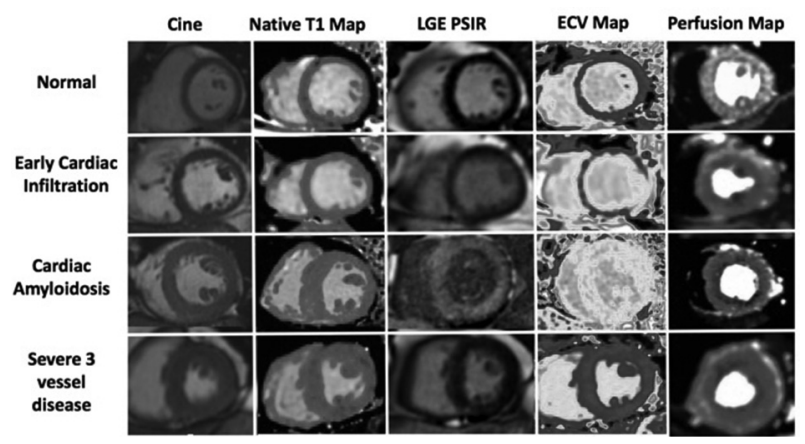

Abstract 19 Figure 1 Short axis cine SSFP images in end-diastole, corresponding native T1 mapping, late gadolinium enhancement (LGE) images, ECV Mapping and stress myocardial blood flow mapping in a normal subject, a patient with early cardiac infiltration (raised ECV, no LGE), a patient with cardiac amyloidosis, a patient with severe three vessel coronary disease $\mathrm{p}<0.001$ and $2.29 \pm 0.87, \mathrm{p}=0.014)$ with the degree of reduction being similar only to patients with $3 \mathrm{VD}(1.44 \pm 0.54$, $\mathrm{p}=1.000$ and $1.64 \pm 0.68, \mathrm{p}=1.000$ ) (figure 1). Rest $\mathrm{MBF}$ was also lower in amyloidosis than HV. Cardiac amyloidosis stress MBF and MPR inversely correlated with amyloid burden (ECV, $\mathrm{r}=-0.715, \mathrm{p}<0.001$, transmurality of LGE, $\mathrm{p}<0.01$ ), systolic dysfunction (EF, r $=0.405, \mathrm{p}<0.01)$, and blood biomarkers (NT-proBNP $(\mathrm{r}=-0.678, \mathrm{p}<0.001)$ and Troponin $\mathrm{T}$ $(\mathrm{r}=-0.628, \mathrm{p}<0.001))$. There was a correlation between stress $\mathrm{MBF}$ and native T1 $(\mathrm{r}=-0.588, \mathrm{p}<0.001)$ but not T2 $(p=0.591)$. Stress MBF and MPR were early disease markers, being elevated in patients with early cardiac amyloid infiltration (raised ECV, no LGE, $\mathrm{p}<0.01$ vsHV).

Conclusion Myocardial ischaemia is common in cardiac amyloidosis - with stress $\mathrm{MBF}$ and MPR similar to that of patients with $3 \mathrm{VD}$. The reduction correlates with the degree of amyloid infiltration and markers of adverse prognosis, highlighting the potential role of myocardial ischaemia as a key mechanism in the pathophysiology of cardiac amyloidosis.

\section{ENDOTHELIAL LOSS AS A CAUSE OF IMPAIRED MYOCARDIAL PERFUSION RESERVE ON CMR IN SEVERE AORTIC STENOSIS}

Kenneth Chan, Betty Raman, Joseph Westaby, Sairia Dass, Mario Petrou, Rana Sayeed, Houman Ashrafian, Saul G Myerson, Theodoros D. Karamitsos, Mary Sheppard, Stefan Neubauer, Masliza Mahmod. University of Oxford Centre for Clinical Magnetic Resonance Research (OCMR), Oxford, UK

\subsection{6/heartjnl-2019-BSCMR.20}

Background Impaired myocardial perfusion reserve occurs in pressure overload hypertrophy such as in severe aortic stenosis (AS) despite unobstructed epicardial coronaries. However the pathological mechanisms underlying this are poorly understood. We sought to assess myocardial perfusion reserve in severe AS by stress perfusion cardiovascular magnetic resonance (CMR), and examine the findings in relation to the histological evidence of vascular changes in the myocardium.

Methods Fourteen patients with severe AS and unobstructed epicardial coronaries underwent adenosine stress perfusion CMR before and 6 months after surgical aortic valve replacement (AVR). Myocardial biopsies were obtained during AVR and stained using CD31 +for endothelium, smooth muscle actin (SMA) for smooth muscle, and picrosirius red for

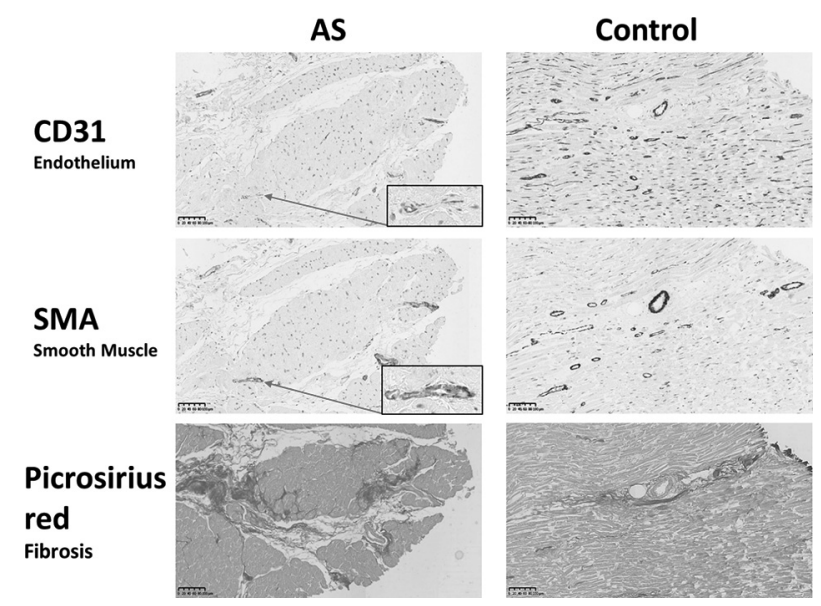

Abstract 20 Figure 1 